artery endothelial cells (iHCAECs) were then integrated within our parallel-plate flow apparatus, in which the response to varying flow conditions was observed.

Results From extensive published and unpublished data, the overexpression of BMI1 enables prolonged cell proliferation whilst inhibiting cell senescence. Utilising lentiviral overexpression, we were able to immortalise HCAEC with BMI1. Isolated iHCAEC clones were placed under varying hemodynamic conditions in order to screen their response. These iHCAEC clones lined up in conjunction with HCAEC counterparts demonstrating a continued mechanosensitivity. Current work investigating gene expression related to the differing hemodynamic environments is underway with full benchmarking against HCAEC controls in progress. TFAR gene constructs have been developed known to regulate atherosclerosis. The TFARs; NFкB, AP-1, IRF3, XBP1, KLF2 and NRF2 have been developed along with lentiviral vectors with secretable luciferase reporters (VLuc and NLuc) allowing for incorporation into validated iHCAECs. Validating these reporter constructs in response to ox-LDL, TNF $\alpha$ and cigarette smoke extract via luciferase activity measurement is currently underway, with selected constructs already cloned.

Conclusion Our initial results indicate the overexpression of BMI1 in HCAEC results in an extended lifespan and inhibition in cell senescence, with morphology unaffected. The resulting iHCAECs exhibit mechanosensitivity to a changing hemodynamic environment comparable to primary HCAECs. This highlights the advantages of this cell line for future CHD modelling, with its integration with our E-Sense system allowing for the development of a novel research tool and the potential to replace pre-existing methods.

Conflict of interest no

\section{Young Investigators Award}

\section{\begin{tabular}{|l|l}
\hline A & ENDOTHELIAL CELL DERIVED EXTRACELLULAR VESICLES
\end{tabular} MEDIATE NEUTROPHIL DEPLOYMENT FROM THE SPLEEN FOLLOWING ACUTE MYOCARDIAL INFARCTION}

\begin{abstract}
${ }^{1}$ Naveed Akbar*, ${ }^{2}$ Alastair Corbin, ${ }^{1,3}$ Sam Dawkins, ${ }^{1}$ Charlotte Lee, ${ }^{1}$ Eleanor Hogg, ${ }^{1}$ Laurienne Edgar, ${ }^{4}$ Mala Gunadasa-Rohling, ${ }^{1}$ Abhirup Banerjee, ${ }^{5}$ Genevieve Melling, ${ }^{6}$ Rebecca Dragovic, ${ }^{3}$ Oxford Acute Myocardial Infarction Study (OxAMI), ${ }^{5}$ Dave Carter, ${ }^{4}$ Paul Riley, ${ }^{2}$ Irina Udalova, 1,3 Keith M Channon, ${ }^{7}$ Daniel Anthony, ${ }^{1,3}$ Robin P Choudhury. ${ }^{1}$ Division of Cardiovascular Medicine, Radcliffe Department of Medicine, University of Oxford; ${ }^{2}$ Kennedy Institute of Rheumatology, University of Oxford; ${ }^{3}$ Acute Vascular Imaging Centre, Radcliffe Department of Medicine, University of Oxford; ${ }^{4}$ Department of Physiology, Anatomy and Genetics, University of Oxford; ${ }^{5}$ Department of Biological and Medical Sciences, Oxford Brookes University; ${ }^{6}$ Department of Women's \& Reproductive Health, University of Oxford; ' Department of Pharmacology, University of Oxford
\end{abstract}

10.1136/heartjnl-2019-BCS.224

Background Acute myocardial infarction (AMI) induces transcriptional activation of monocytes en route to the injured myocardium, in part driven by endothelial cell derived extracellular vesicles (EC-EV), which contain proteins and microRNA (miRNA) cargo. However, neutrophils are the first immune cells to arrive at sites of injury and mediate further damage to the ischemic myocardium. Here, we describe for the first time how neutrophils are released from the spleen in AMI and show that this is driven by EC-EV signalling.

Methods and results Experimental AMI in wild-type mice caused a significant increase in peripheral blood neutrophils and a simultaneous reduction in splenic-neutrophil number $(\mathrm{P}<0.01)$, suggesting splenic-neutrophil deployment, which is a previously unknown neutrophil reserve in AMI. Patients have elevated peripheral blood neutrophil (1.6-fold, $\mathrm{P}<0.01)$ and plasma EV numbers $(2.2$-fold, $\mathrm{P}<0.01)$ at the time of AMI presentation, which significantly correlate $(\mathrm{R}=0.29, \quad \mathrm{P}=0.037)$ and suggests plasma EV-neutrophil interactions. EC-EV can alter immune cell motility from the spleen (Akbar et al, 2017). Patient plasma EV (isolated by differential ultracentrifugation, EV confirmed by protein markers TSG101, ALIX, CD9, HSP70 and morphology by transmission electron microscopy) show enrichment for ECvascular cell adhesion molecule-1 (VCAM-1) and ECmiRNA-126-3p. AMI induces EC activation; EC activation with pro-inflammatory TNF- $\alpha$ models this in vitro, causing increased $\mathrm{EC}-\mathrm{EV}$ release $(\mathrm{P}<0.001)$ and enrichment for miRNA-126-3p $(\mathrm{P}<0.01)$. EC-miRNA-126 is a negative regulator of EC activation and may dually control EC-EV release. CRISPR-edited-miRNA-126 knock-out EC display a pro-inflammatory phenotype, as evidenced by increased VCAM-1 $(\mathrm{P}<0.001)$ expression and show enhanced EC-EV release $(\mathrm{P}<0.001)$. To better understand the potential role of miRNA-126 on neutrophil biology we analysed miRNA126-putative-mRNA targets and compared these to neutrophil Gene Ontology (GO) pathway terms. miRNA-126mRNA targets are significantly over represented when compared to neutrophil GO terms for: degranulation $(\mathrm{P}<0.001)$, activation $(\mathrm{P}<0.001)$, chemotaxis $(\mathrm{P}=0.008)$ and migration $(\mathrm{P}=0.008)$. EC-EV exposure to primary human neutrophils alters inflammatory IL-6 $(\mathrm{P}<0.01)$ and chemokine gene expression (CCL7 $(\mathrm{P}<0.01)$ and CCL18 $(\mathrm{P}<0.05))$, substantiating bioinformatic findings. EC-EV tail vein injected into wild-type, naive mice mobilise splenicneutrophils to peripheral blood $(\mathrm{P}<0.001)$, confirming splenic neutrophil mobilisation by EC-EV.

Conclusions (I) Neutrophil deployment from the spleen is a novel finding in acute injury and interactions with (II) EC-EV may mediate their splenic liberation and (III) activation following AMI, en route to the injured myocardium. The splenic neutrophil reserve may be a novel therapeutic target in AMI to modulate the inflammatory response before recruitment of cells to sites of injury. 